\title{
Perelman, argument ad hominem et ethos rhétorique
}

Perelman, ad Hominem Argument, and Rhetorical Ethos

\section{Michael Leff}

Traducteur : Sivan Cohen-Wiesenfeld

\section{CpenEdition Journals}

\section{Édition électronique}

URL : http://journals.openedition.org/aad/213

DOI : 10.4000/aad.213

ISSN : 1565-8961

\section{Éditeur}

Université de Tel-Aviv

\section{Référence électronique}

Michael Leff, «Perelman, argument ad hominem et ethos rhétorique », Argumentation et Analyse du Discours [En ligne], 2 | 2009, mis en ligne le 01 avril 2009, consulté le 23 septembre 2019. URL : http:// journals.openedition.org/aad/213; DOI : 10.4000/aad.213

Ce document a été généré automatiquement le 23 septembre 2019.

\section{cc) (†)}

Argumentation \& analyse du discours est mis à disposition selon les termes de la licence Creative Commons Attribution - Pas d'Utilisation Commerciale - Pas de Modification 4.0 International. 


\title{
Perelman, argument ad hominem et ethos rhétorique
}

Perelman, ad Hominem Argument, and Rhetorical Ethos

\author{
Michael Leff
}

Traduction : Sivan Cohen-Wiesenfeld

\section{NOTE DE L'ÉDITEUR}

Ce texte est la version française d'un article publié dans Argumentation $2009: 2$, « Perelman and Beyond. Current Issues in Argumentation Studies ", Amossy, Koren and Yanoshevsky (eds). Traduit de l'anglais par Sivan Cohen-Wiesenfeld.

1 Au cours du demi-siècle qui a suivi la publication de la Nouvelle Rhétorique de Perelman et Olbrechts-Tyteca, l'attitude manifestée envers la rhétorique, l'argumentation, et le rapport qui se noue entre elles, a connu un changement spectaculaire. Prenons, par exemple, la première phrase de la Nouvelle rhétorique: "La publication d'un traité consacré à l'argumentation et son rattachement à une vieille tradition, celle de la rhétorique et de la dialectique grecques, constituent une rupture avec une conception de la raison et du raisonnement, issue de Descartes, qui a marqué de son sceau la philosophie occidentale des trois derniers siècles" (1992: 1). La force de cette affirmation sans ambiguïté n'échappe aujourd'hui à personne, mais il est nécessaire, pour comprendre à quel point elle était radicale lors de la parution de l'ouvrage, de se replacer dans son contexte historique. La révolution contre Descartes et ses compagnons des Lumières avait alors à peine commencé. De nos jours, bien que la rhétorique n'ait pas encore perdu toutes ses connotations péjoratives, des chercheurs réputés l'invoquent régulièrement sans causer pour autant de scandale. Ce n'était pas le cas il y a cinquante ans, et la pensée qu'un philosophe respectable puisse répudier l'héritage cartésien et sympathiser avec des rhétoriciens (fût-ce des rhétoriciens grecs) paraissait alors non moins scandaleuse que l'idée de Mamie Eisenhower, l'épouse 
exemplaire du président des Etats-Unis, maculant ses gants blancs, divorçant de son mari et rejoignant un gang de hors-la-loi à moto.

2 Si la Nouvelle rhétorique a été le précurseur d'une grande partie des développements majeurs qui ont vu le jour, depuis sa publication, dans les domaines de la rhétorique et de la logique informelle, c'est précisément parce qu'elle a constitué une cassure si brutale et si systématique avec la conception rationnelle des Lumières. L'un des aspects les plus importants de ce changement, que je souhaite développer ici, concerne le rôle de la personne dans l'argumentation. Les différences essentielles qui se font jour sur ce point sont symptomatiques d'approches fondamentalement différentes de l'argumentation.

3 Le paradigme rationaliste a tenté de minimiser les considérations personnelles dans l'argumentation, voire de les éliminer totalement. Dans l'optique du traitement dit «standard» des paralogismes, les arguments sont supposés se rapporter uniquement au contenu des assertions et aux relations logiques entre elles. Toute référence ou appel à un élément étranger à ces considérations détourne de la délibération objective et gêne la clarté du raisonnement. Parmi ces déviations, les principales sont constituées par les facteurs liés aux personnes engagées dans le débat, que ce soit à leur caractère, à leur comportement ou à leurs prises de positions passées. L'intrusion de telles considérations personnelles est considérée comme non pertinente et à l'origine d'une forme de raisonnement invalide désigné comme le paralogisme de l'argument ad hominem ${ }^{1}$.

4 Perelman adopte une perspective entièrement différente. Sa théorie de l'argumentation s'attache à rechercher comment "provoquer ou accroître l'adhésion des esprits aux thèses qu'on présente à leur assentiment » (1992:5), processus qui ne peut se dérouler dans un espace dépersonnalisé et qui requiert la participation des individus qui avancent des arguments ou y répondent. Perelman renverse donc la doctrine rationaliste. A l'inverse des théories qui refusent fermement de reconnaître l'importance de l'auditoire, la conception de l'argumentation de Perelman est «toute entière relative à l'auditoire qu'elle cherche à influencer » (24). La Nouvelle rhétorique insiste de façon répétée et catégorique sur ce point : «C'est à cause des rapports qu'elle possède avec l'action, parce que l'argumentation ne se déroule pas dans le vide, mais dans une situation socialement et psychologiquement déterminée, qu'elle engage pratiquement ceux qui y participent» (78), et donc l'objectivité oblige à une réinterprétation afin de reconnaître l'impossibilité de séparer « une affirmation de la personne de celui qui la pose » (78). Dans un passage que j'étudierai plus loin en détail, Perelman affirme que «cette interaction entre orateur et discours serait même la caractéristique de l'argumentation, par opposition à la démonstration » (426).

5 La pensée de Perelman sur ce sujet le situe très en avance sur son temps, et au fil des décennies, le rôle des personnes dans l'argumentation est devenu l'objet d'une attention accrue dans plusieurs disciplines, ainsi que dans un cadre interdisciplinaire. Cependant, l'étude la plus approfondie sur ce sujet a été menée par des spécialistes de logique informelle et de dialectique, courants qui s'attachent à repenser la conception de l'argument ad hominem.

6 Alors que Perelman se contente de rejeter implicitement le traitement standard de l'argument ad hominem, les dialecticiens sont beaucoup plus nets et explicites, voire même abrupts, dans leur critique. Reprenant à leur compte l'impitoyable verdict de C. L. Hamblin qui le qualifie de «dégradé, dépassé et dogmatique »², ils expriment leur 
indignation chaque fois qu'ils en retrouvent la trace dans les pages d'un manuel de logique et le posent comme une effigie antagoniste face à laquelle ils définissent leurs efforts pour réformer et élargir le domaine de l'argumentation. Ces reproches découlent d'un refus global de la tradition logico-formelle et reflètent l'idée selon laquelle les arguments "dépendent à juste titre d'éléments allant au-delà de leur contenu propositionnel » (Gouvier 1999: 20). A cet égard, les dialecticiens s'accordent entre eux, ainsi qu'avec Perelman, pour penser que la théorie de l'argumentation doit prendre en compte les personnes.

7 Ces points d'accord ont cependant leurs limites, et les choses se compliquent lorsque l'on en vient à la question des moyens et des buts sur lesquels les chercheurs doivent faire porter leurs efforts pour « repeupler » les études d'argumentation. Les spécialistes de logique informelle et les dialecticiens se divisent alors en plusieurs camps suivant leur point de vue sur l'argument ad hominem, et trois positions distinctes émergent. La première présente une justification minimaliste de cet argument, basée sur une distinction entre les aspects propositionnel et situationnel de l'argumentation. Dans cette optique, une attaque ad hominem n'a pas nécessairement de conséquence sur la vérité d'une affirmation, mais peut, et parfois doit, limiter la portée d'une proposition exprimée par une personne spécifique. Ainsi, par exemple, si un maire prône une réduction de salaire pour tous les employés de la municipalité tout en augmentant ses propres émoluments, l'incohérence de son comportement ne préjuge en rien du bienfondé de la diminution salariale, mais le discrédite en tant que défenseur de cette proposition (ibid.).

8 La seconde approche se situe dans le cadre de la révision globale de la théorie des arguments fallacieux par l'école de la pragma-dialectique, et implique le déplacement de l'intérêt des formes valides du raisonnement vers le souci des normes qui président à la discussion perçue comme interaction sociale. Dans cette perspective, un argument fallacieux est un « raté » du processus de communication qui empêche la résolution de la controverse, et l'attaque ad hominem est un argument fallacieux par lequel un énonciateur viole l'une des normes pragma-dialectiques en attaquant la personne d'un autre interlocuteur réel ou potentiel, en tentant, par exemple, de l'embarrasser afin de l'empêcher d'exprimer un point de vue pertinent sur le sujet en question ${ }^{3}$. Enfin, Douglas Walton tente de se débarrasser du terme de "fallacie» appliqué aux arguments énumérés dans le traitement standard et propose de concevoir ceux-ci comme des schèmes argumentatifs, qui peuvent être fallacieux ou légitimes selon la façon dont ils sont utilisés. Dans un long traité entièrement consacré aux arguments ad hominem, Walton met au point une typologie complexe des schèmes ad hominem et propose des normes pour les évaluer (Walton 1998).

9 Les différences entre ces positions sont significatives, la seconde et la troisième provenant d'un programme complexe d'études en argumentation comprenant d'importantes considérations rhétoriques. Toutes trois ont cependant un «air de famille » qui dévoile une paternité dialectique commune. La nature de cette affinité et sa signification émergent lorsqu'on compare les orientations dialectique et rhétorique qui se rapportent au traitement des personnes dans l'argumentation. Cette comparaison donne en même temps un aperçu de la pensée de Perelman et fait émerger les questions que soulève sa théorie, qui se construit à la frontière de la dialectique et de la rhétorique. 
10 Alan Brinton $(1985,1986,1995)$ et Ruth Amossy $(2001: 1-23)$ notent tous deux que l' ethos rhétorique est la contrepartie de l'argument ad hominem en dialectique, et en reprenant et élargissant leurs analyses, on peut établir une comparaison qui fait apparaitre quatre différences principales entre ces deux conceptions jumelles :

11 (1) L'argument ad hominem dialectique relève du négatif et se réfère aux commandements " $\mathrm{Tu}$ ne feras point» de l'argumentation. Il désigne les bévues argumentatives que les locuteurs doivent éviter et que les récepteurs doivent être capables d'identifier et de dévoiler. L'ethos rhétorique fonctionne comme une ressource créatrice. Il offre la possibilité aux locuteurs de construire des images de soi positives, des représentations négatives de leurs opposants, et de mettre en œuvre tout autre moyen relatif aux personnes susceptible de les aider à atteindre leur objectif.

(2) Les dialecticiens contemporains considèrent l'argument ad hominem comme faisant partie d'une interaction sociale, mais ils limitent celle-ci à l'échange argumentatif en soi et à ses assertions explicites. Les aspects plus larges du contexte, liés aux questions sociales, culturelles ou intertextuelles, sont évacués. Ces derniers sont, au contraire, cruciaux pour la rhétorique, puisque, comme le dit Brinton, l'objectif du locuteur est de refléter "l'ethos général de la société"; c'est ainsi qu'il cherche à "exprimer nos valeurs partagées, penser en termes de prémisses communes, exercer à bon escient son jugement et parler pour nous » (Brinton $1985: 55$ ).

13 (3) Les dialecticiens évaluent la crédibilité et l'autorité en rapport avec la pertinence et la force des assertions émises sur les personnes. Les rhétoriciens considèrent à la fois ce qui est dit et la façon dont on le dit, et étudient souvent la crédibilité (ou son opposé) comme résultant de la construction de traits ou de types de caractères à travers la performance discursive.

14 (4) L'objectif de la dialectique est l'établissement de critères généraux permettant d'évaluer le caractère justifié ou non d'une croyance ou des processus susceptibles de résoudre une controverse. Les études de dialectique gravitent donc autour de la recherche de catégories abstraites ou de structures argumentatives. Des efforts considérables sont ainsi réalisés en vue de définir les caractéristiques des arguments ad hominem, de les subdiviser en sous-types (attaque directe, circonstancielle, argument tu quoque etc.) et de définir des normes permettant d'évaluer ces variantes telles qu'elles apparaissent au cours des différentes phases de l'argumentation ou dans des discussions ayant des objectifs différents (phases et objectifs étant décrits dans des schèmes abstraits). L'objectif de la rhétorique est de développer la capacité à utiliser les arguments et à en comprendre le fonctionnement. Les rhétoriciens ont donc tendance à se référer aux catégories et aux structures de façon pragmatique, en les adaptant à leurs objectifs en fonction des cas particuliers et des auditoires spécifiques.

Perelman, on le sait, a très consciemment choisi le terme de rhétorique et non celui de dialectique pour désigner son projet, et ce en partie en raison des différences rappelées plus haut entre les deux domaines, qui font ressortir l'importance accordée par la rhétorique au contexte de l'argumentation, et à son lien avec l'auditoire. Dans le passage cité plus haut, Perelman indique très clairement qu'il considère l'argumentation comme étant liée à une action située dans un contexte social et psychologique déterminé, entraînant de ce fait une interaction entre les parties impliquées dans le débat ainsi qu'un engagement de leur part, et que donc, pour lui, les thèses d'un argumentateur ne peuvent être détachées de sa personne. A cet égard, non 
seulement il rompt définitivement avec le modèle formaliste, mais il repousse aussi les limites de la logique informelle et de la dialectique ${ }^{4}$.

Pourtant, la Nouvelle rhétorique ne fournit pas l'analyse dense et nuancée du rôle des personnes dans l'argumentation à laquelle on aurait pu s'attendre au vu des positions de Perelman. Il n'est fait qu'une référence brève et incidente au concept d'ethos, et l'analyse détaillée de l'acte et de la personne dans l'argumentation (Partie III, sections 68 à 73) ressemble plus à un inventaire de tactiques qu'à une théorie sur la manière dont l'argument est lié à la personne de l'argumentateur et en fait usage. La clé de cette anomalie se trouve, me semble-t-il, dans la section consacrée au « discours comme acte du locuteur» (III, 72) sur laquelle je vais me pencher de plus près. Auparavant, je remarquerai toutefois que Perelman traite de la question de l'argument ad hominem dans une section intéressante et dense, située vers la fin du paragraphe consacré à «l'accord » comme point de départ de l'argumentation (II, 28: 148-153).

Perelman constate que, selon sa conception de l'argumentation, tous les arguments sont soit ex concessis soit ad hominem. Contrairement aux logiciens, qui distinguent entre les arguments ad hominem, basés sur la personne, et les arguments ad rem, fondés sur les faits, Perelman considère ces derniers comme de simples points d'accord faisant référence à un auditoire universel. Ce que les autres considèrent comme des prémisses ad rem est donc, pour lui, uniquement une catégorie particulière d'hypothèses établies à partir de l'accord de l'auditoire. De plus, dans la mesure où les arguments orientés vers l'auditoire universel prétendent être valides pour tous les êtres rationnels, Perelman suggère de les nommer ad humanitatem et de les considérer comme une classe particulière d'arguments ad hominem. Mais il modifie presque immédiatement cette distinction en proposant une approche de l'argument ad hominem « au sens étroit » qui ne s'appliquerait qu'aux cas dans lesquels les locuteurs avancent des arguments qu'ils savent «sans poids face à l'auditoire universel». Le concept est déroutant, mais Perelman n'en n'offre pas d'explication excepté un court exemple, et conclut le débat par une nouvelle distinction, séparant cette fois l'argument ad hominem de l'argument ad personam, le premier se rapportant à la cohérence ou à l'incohérence des croyances d'un auditoire particulier, alors que le second concerne une attaque personnelle visant à discréditer un opposant.

18 Ces observations me paraissent à la fois riches en suggestions et déconcertantes. Perelman déverse sur près de deux pages une accumulation de notions, dont certaines présentent un changement radical par rapport aux définitions et aux conceptions habituelles, suggérant, d'une manière déroutante, de reconsidérer l'ensemble des attaques ad hominem et d'en distinguer les incarnations rhétoriques de leurs manifestations sous d'autres formes. Mais, dans la mesure où il ne concrétise de façon substantielle aucune de ces manœuvres terminologiques, la discussion semble accessoire, et sans lien apparent avec l'analyse de la place de l'acte et de la personne dans l'argumentation qu'il développe plus loin.

Celle-ci apparaît dans la troisième partie et s'étend sur trente-cinq pages, subdivisées en six sous-chapitres. Le chapitre tout entier mériterait d'être soigneusement étudié par quiconque s'intéresse à l'argumentation fondée sur le caractère et le statut des argumentateurs, mais je ne traiterai ici que l'un de ses sous-chapitres, «Le discours comme acte du locuteur» (section 72). C'est ici que Perelman touche le plus directement au concept d'ethos, qu'il y fait explicitement référence. 
20 Le paragraphe débute en mettant en évidence l'interaction entre orateur et discours, affirmant que celle-ci serait la caractéristique même de "l'argumentation, par opposition à la démonstration » (426). Il s'agit d'une affirmation forte, même si elle est tempérée par l'adverbe "peut-être ", et on s'attend à quelques développements ou justifications. A la place de ceux-ci, cependant, Perelman se livre, comme partout ailleurs dans le traité, à un inventaire de techniques et de stratégies argumentatives en fournissant des conseils sur leur utilisation. Certaines se réfèrent aux techniques courantes de l'argumentation «ethotique ", comme le fait de conquérir le respect, la bienveillance et la sympathie de l'auditoire et les efforts correspondants pour discréditer la personnalité de ses adversaires. Mais certains de ces points portent plutôt sur des considérations spécifiques relevant du domaine du performatif : par exemple le fait que la qualité de l'argumentation du locuteur influence la perception que l'auditoire a de lui; que le locuteur doit se concilier l'auditoire en montrant qu'il a confiance dans son jugement ; qu'il doit souvent agir comme un mentor, prenant soin de ne pas susciter d'hostilité en traitant son auditoire comme s'il était inférieur; enfin tenir compte de ses auditeurs en adoptant une attitude retenue et discrète, éviter d'étaler ses connaissances de façon arrogante et rester bref. Toutes ces questions font référence à la conduite de l'argumentateur en situation et à l'interaction qu'il noue avec son auditoire spécifique, et elles indiquent les limites de l'analyse logique ou des normes abstraites comme critère d'évaluation des arguments liés à l'ethos dans la mesure où ni les propositions, ni les normes abstraites, ne peuvent rendre compte de la façon dont le locuteur établit son rapport avec l'auditoire dans un cas particulier. Ces observations sur le style et le comportement du locuteur sont donc en relation directe avec l'affirmation de Perelman selon laquelle l'interaction constitue la distinction cruciale entre le domaine de l'argumentation et celui de la logique formelle, et contribuent à la conforter. L'auteur ne développe cependant aucun de ces points, il n'y apporte aucune illustration ni analyse approfondie, et ne les relie pas à son point de départ théorique. Pour quelle raison?

21 Cette attitude s'explique sans aucun doute en partie par le plan de la Nouvelle rhétorique, qui traverse des champs de connaissances rhétoriques si étendus qu'il laisse peu de place à l'approfondissement. Une autre raison, moins évidente mais peut-être plus intéressante, apparait cependant lorsque l'on examine le passage où Perelman mentionne explicitement le concept d'ethos et le définit: "Ce que les anciens avaient l'habitude d'appeler ethos rhétorique peut être résumé comme l'impression que le locuteur donne de lui-même à travers ses mots. Isocrate dit ceci : "Ne soutenez jamais une mauvaise cause, car les gens vous suspecterons de faire vous-même les choses que vous aidez les autres à faire" " (319). Ce passage n'est pas sans produire quelque confusion, notamment parce qu'il lie deux conceptions totalement différentes de l'ethos : l'une aristotélicienne, qui le présente comme un mode de preuve entièrement limité aux paroles prononcées par l'énonciateur, l'autre isocratéenne, qui prend en compte la réputation du locuteur établie sur des paroles et des faits antérieurs au discours.

Au-delà de la confusion spécifique et technique qui peut facilement être clarifiée, le passage dévoile un problème beaucoup plus fondamental concernant la compréhension de la tradition rhétorique. Perelman, dans ce cas comme partout ailleurs, considère celle-ci comme un ensemble unifié, conception relativement courante mais pourtant inexacte. La rhétorique classique comprend plusieurs variantes, dont les deux plus marquantes sont représentées, d'un côté par Aristote, et de l'autre par une tradition 
qui s'étend d'Isocrate à Cicéron et Quintilien, puis aux humanistes de la Renaissance ${ }^{5}$. Aristote met l'accent sur le statut de la rhétorique comme art et la conçoit comme un ensemble de préceptes, organisés sur un mode plus ou moins systématique et orienté vers la recherche de moyens de persuasion. L'autre position, que Janet Atwill (1998) nomme avec justesse la "tradition de l'éloquence", met en avant la personne de l'orateur, considère "l'art» rhétorique comme une partie, utile mais auxiliaire, de l'entreprise, et prend comme objectif le développement des capacités d'adaptation qui permettent de faire face adéquatement aux circonstances de chaque cas particulier.

La différence globale entre ces perspectives détermine leurs différentes conceptions de l'argumentation "ethotique ». Les aristotéliciens relèvent les techniques qu'un locuteur peut utiliser dans la construction de l'ethos d'un personnage, mais font l'impasse sur des questions contextuelles comme la réputation préalable parce qu'elles sont extrinsèques à l'art. Les isocratéens, par contre, cherchent à comprendre comme on représente un personnage dans chaque cas particulier, les questions extrinsèques, comme la réputation préalable, faisant alors nécessairement partie des données de départ. Ils considèrent la connaissance des techniques comme moins édifiante que la pratique et l'étude des modèles. Perelman est sensible aux dimensions sociales et culturelles de l'argumentation, et comme nous venons de le remarquer, il reconnait ouvertement que la mise en œuvre est d'une importance cruciale dans le processus argumentatif. Cependant, son attitude est dans l'ensemble aristotélicienne. Il explique dans son introduction de la Nouvelle rhétorique que son projet « se préoccupant surtout de la structure de l'argumentation " n'insiste pas «sur la manière dont s'effectue la communication avec l'auditoire» (1992: 8). Et de fait, l'ouvrage relève et explicite méthodiquement les structures et les techniques argumentatives, et ce de façon très productive, mais ne fournit pas de descriptions en profondeur de la pratique rhétorique, ni ne montre le fonctionnement des techniques argumentatives à l'intérieur du processus global de persuasion. Comme dans un traité dialectique, les exemples, même s'ils sont développés, sont utilisés pour illustrer des principes plus que comme modèle d'imitation ou comme stimulateur d'une approche heuristique.

Etant donné sa conception de l'argumentation comme acte en situation, il aurait pu sembler important pour Perelman d'enrichir son schéma aristotélicien d'un intérêt isocratéen pour le fonctionnement en acte de l'argumentation. Qu'il ne l'ait pas fait creuse un fossé entre sa conception de la tradition rhétorique et la représentation qu'en donne la Nouvelle Rhétorique. Sans doute ne s'agit-il pas d'un échec à réaliser la tâche essentielle qu'il s'était fixée, à savoir traiter des techniques de l'argumentation rhétorique de façon exhaustive et tenue à jour. Il s'agit bien plutôt d'une incapacité à reconnaître que les techniques ne fournissent qu'une partie des éléments nécessaires à la compréhension du fonctionnement des arguments. Cinquante ans après Perelman, je pense qu'il serait extrêmement utile de développer son projet en s'attaquant à des cas de figures, et c'est ce que je me propose de faire pour conclure cet article.

Ruth Amossy, en se basant sur sa propre étude de cas, a expliqué que l'un des problèmes-clés concernant l'ethos est d'arriver à déterminer qui possède l'autorité à parler dans des circonstances spécifiques, ou, pour reprendre sa métaphore empruntée à Bourdieu, qui a le droit de tenir le sceptre (2001 : 3). L'exemple que j'ai choisi pose ce problème d'une façon particulièrement aiguë, puisqu'il concerne un écrivain appartenant à une classe marginalisée, qui utilise des arguments «ethotiques » pour 
trouver un ton qui reste distinctif tout en étant suffisamment adapté à la culture dominante pour bénéficier d'un auditoire.

Dans son essai « Booker T. Washington et autres» (publié en 1903 comme un chapitre des Ames du peuple noir), W. E. B. DuBois tente de définir un programme politique pour les Afro-Américains à un moment troublé de leur histoire. Soumis à la loi raciste "Jim Crow » dans le sud et à l'exploitation économique et sociale systématique à travers le pays, les Afro-Américains se voyaient refuser une place significative au sein du débat public et étaient virtuellement exclus du pouvoir politique. Seul l'un d'entre eux, Booker $\mathrm{T}$. Washington, parvint à gagner la reconnaissance de l'establishment blanc et à se faire entendre à travers la ligne de démarcation raciale. Cependant, il employait une rhétorique si parfaitement assimilée au langage dominant du monde blanc qu'elle semblait ne pas s'en distinguer.

DuBois trouva l'influence de Washington restrictive, et chercha à énoncer un message plus fort, plus indépendant et plus critique, à la fois destiné à la communauté noire et émanant d'elle. Pour ce faire, il devait néanmoins trouver un moyen rhétorique permettant d'exprimer la dissidence afro-américaine dans une sphère publique qui n'offrait pas encore de scène à celle-ci. Dans son essai, il chercha à résoudre ce problème en utilisant l'ethos contraignant mais autoritaire de Washington, tout en se démarquant de lui. Les trois premières phrases de cet essai annoncent avec subtilité le but et la stratégie de DuBois :

Sans nul doute, la chose la plus marquante de l'histoire des Noirs américains depuis 1875 est l'influence de M. Booker T. Washington. Elle commença à une époque où les souvenirs de guerre et les idéaux étaient en voie de disparition rapide; l'aube d'un étonnant développement commercial se levait; un sentiment de doute et d'hésitation submergeait les fils de la liberté, ce fut alors que vint son heure. M. Washington arriva avec un programme unique bien défini, au moment psychologique où la nation avait un peu honte d'avoir investi tant de sentiment dans les Nègres, et concentrait à présent ses énergies sur les Dollars. (DuBois. 1989 : 30)

La phrase d'ouverture met en place une voix narrative off de l'auteur qui prend ses distances par rapport aux diversions momentanées et adopte une perspective historique large ; le style est impersonnel, académique, celui du diagnostic clinique. De son observatoire, ce qui frappe DuBois n'est pas le rôle de Washington comme agent, comme dirigeant actif, mais sa position inerte dans le paysage de l'histoire; son émergence n'est même pas un évènement, mais une "chose ». La construction impersonnelle et indirecte de la phrase suggère fortement la passivité de Washington. Imaginez quelle aurait été cette force, intégrée dans une syntaxe plus active, et peutêtre plus normale, par exemple : «L'influence de M. Booker T. Washington est sans nul doute la chose la plus marquante de l'histoire des Noirs américains depuis 1876 ». Dans la version de DuBois, par contraste, le nom de Washington apparaît seulement à la fin de la phrase, isolé du verbe par une préposition, et son influence semble venir moins d'une personne active dans l'histoire que d'un phénomène qui s'est simplement produit dans l'histoire. Enfin, le cadre temporel utilisé par DuBois est également signifiant sur le plan rhétorique. Washington est replacé dans le contexte des événements qui se sont produits depuis 1876, c'est-à-dire depuis la fin de la Reconstruction et l'avènement de Jim Crow, période sombre dans l'histoire des Afro-Américains. L'influence de Washington correspond donc à une période de régression dans la destinée de son peuple. 
29 En laissant pour le moment de côté la deuxième phrase, nous remarquons que la troisième présente Washington comme un agent plus actif. Son nom y apparaît au début de la phrase et dans une position nominative. Mais l'accent est mis, non sur ce que Washington a fait, mais sur le moment où il est apparu sur la scène. Il se trouva simplement au bon endroit au bon moment, et ne s'engagea dans une action indépendante que pour " promouvoir un programme unique et bien défini » adapté aux circonstances. Washington reste donc une figure essentiellement réactive, dont le programme s'ajuste aux forces extérieures sans aucun effort pour y résister ni les modifier.

30 Ces deux phrases anticipent plusieurs thèmes-clés qui persistent tout au long de l'essai, selon lesquels le programme de Washington est une adaptation passive aux circonstances extérieures, son leadership trouve ses racines dans des sources extérieures à la communauté afro-américaine, il est extrêmement limité et obstiné, et présente un aspect résolument régressif.

31 La deuxième phrase suggère également certains de ces thèmes, mais son caractère le plus marquant en est la syntaxe alambiquée ; l'ordre des mots est manipulé de façon à ce que la phrase toute entière soit circonscrite par le verbe « commencer » qui se réfère au leadership de Washington. Cette phrase longue et complexe retourne finalement vers son point de départ, car la présence passive mais inévitable de Washington la délimite à chaque extrémité. La structure de cette phrase préfigure la stratégie de DuBois dans l'essai, qui tourne autour de la présence hégémonique de Washington et se développe autour du motif d'un piège qui ne peut être déjoué que par la dissidence critique.

L'articulation de cette stratégie est beaucoup trop détaillée et nuancée pour être correctement synthétisée dans cet article. Cependant, ses principales tendances peuvent être résumées comme suit : l'autorité de Washington est actuellement si forte et si immuable qu'elle est devenue une puissance monolithique réduisant toute critique au silence. Cependant, la critique est l'essence même de la démocratie, aussi est-ce le rôle des Afro-Américains de rechercher d'autres dirigeants pour prendre la parole, ouvrir le débat public et exprimer leurs différences avec Washington. Et dans la mesure où les vues de celui-ci sont extrêmement proches de celles de la culture blanche dominante, les Afro-Américains, en les critiquant, entrent par là-même dans un débat sur les idées qui circulent dans la doxa politique américaine. Par conséquent, la critique de Washington conduit les Afro-Américains à traverser le «voile » qui les sépare de la communauté blanche environnante, et cette activité les dirige également vers un forum de débat politique d'une portée véritablement nationale. Ainsi, non seulement ils y gagnent du pouvoir, mais ils revigorent aussi le système politique américain, dont la santé dépend du libre exercice de la critique et de l'opposition. Bref, DuBois réclame le sceptre en utilisant et en mettant en œuvre des thèmes rhétoriques que les Américains reconnaissent comme des composantes essentielles de leur culture politique, en commençant par sa propre communauté, et en se construisant un ethos auctorial de promoteur actif des intérêts afro-américains contrastant avec l'ethos passif, et donc essentiellement contre-productif, qu'il attribue à Washington ${ }^{6}$.

33 Au cours de cet essai, DuBois déploie de nombreuses techniques argumentatives décrites dans la Nouvelle rhétorique, et si j'avais suffisamment de temps et mes lecteurs assez de patience, je pourrais travailler le texte pour découvrir leur présence et apprécier comme ils fonctionnent dans le cadre du métabolisme de la rhétorique de 
DuBois. Cet exercice améliorerait certainement notre compréhension de l'essai et développerait sans doute notre connaissance de la rhétorique et de l'argumentation, car il nous permettrait de discerner des aspects de la mise en œuvre des arguments qui peuvent difficilement être appréhendés en dehors du contexte. Quoiqu'il en soit, l'appréhension de la fonction argumentative de la personne de l'auteur (de l'interaction persuasive entre le «locuteur » et le "discours ») repose sur une analyse fine du texte et une compréhension en profondeur de son contexte. La connaissance des techniques argumentatives constitue une ressource utile pour l'investigation rhétorique, mais est insuffisante en elle-même pour rendre compte des pratiques spécifiques des argumentateurs.

Pour exprimer ce point en des termes différents et plus généraux, j'affirmerai qu'il existe une tension fondamentale à l'intérieur du projet de Perelman. D'une part, il adopte une perspective fondamentalement rhétorique sur l'argumentation qu'il considère comme un acte en situation, mais de l'autre son analyse repose sur un système orienté dans une perspective dialectique d'identification et de classification de techniques argumentatives abstraites et décontextualisées. Peut-être cette tension apparaît-elle dès la phrase d'ouverture de la Nouvelle rhétorique, où Perelman et Olbrechts-Tyteca relient leur traité «à l'ancienne tradition de la rhétorique et de la dialectiques grecques». Cette formulation suggère une fusion de deux approches de l'argumentation étroitement liées mais cependant distinctes, et il serait probablement utile pour nous de comprendre cette distinction telle qu'elle est suggérée dans le corps de la Nouvelle rhétorique et illustrée dans les recherches des nouveaux dialecticiens et spécialistes de la logique informelle. La tendance dialectique fournit un inventaire des stratégies argumentatives et des grandes lignes de leurs conditions d'applications particulières, offrant par là un guide global de la gamme gigantesque, divergente et souvent déconcertante des pratiques qui entrent dans la mise en œuvre de la rhétorique. La rhétorique et la dialectique sont donc en position de complémentarité mutuelle : la dialectique donne forme à l'investigation rhétorique, tandis que celle-ci empêche les abstractions dialectiques de parvenir au point où elles perdent contact avec la réalité argumentative. Vue sous cet angle, la tension contenue dans la Nouvelle rhétorique fait beaucoup plus que mettre en évidence une contradiction à résoudre. Elle suggère que la rhétorique et la dialectique sont des arts jumeaux (des antistrophoi, pour reprendre le terme d'Aristote) qui doivent interagir. Cette mise en relation peut définir la dialectique comme art d'argumenter face à la rhétorique, art de persuader, ou bien considérer ces deux domaines comme des catégories de l'argumentation. Mais dans les deux cas, il faut reconnaître la nécessité de s'intéresser à la fois à l'arsenal des stratégies argumentatives et aux cas particuliers dans le cadre desquels elles sont mises en œuvre au cours du processus argumentatif. 


\section{BIBLIOGRAPHIE}

Amossy, Ruth. 1999. « The argument ad hominem in an interactional perspective ", Proceedings of the Fourth International Conference on Argumentation (Amsterdam : Sic Sat), 14-18

Amossy, Ruth. 2001. «Ethos at the Crossroads of Disciplines: Rhetoric, Pragmatics, Sociology ", Poetics Today 22, 1-23

Atwill, Janet. 1998. Rhetoric Reclaimed : Aristotle and the Liberal Arts Tradition (Ithaca : Cornell University Press)

Brinton, Alan. 1985.« A Rhetorical View of ad Hominem », Australian Journal of Philosophy 63, 50-63

Brinton, Alan. 1986. « Ethotic Argument », History of Philosophy Quarterly 3, 255-258

Brinton, Alan. 1995. « The ad Hominem », Hansen \& Pinto, 213-222

Conley, Thomas M. 1990. Rhetoric in the European Tradition (White Plains, NY : Longmans), 4-25

DuBois, W. E. B. 1989 [1903]. Souls of the Black Folk (New York : Bantam)

Gouvier, Trudy. 1999. "Political Speech, Oliver Sachs and the Credibility Concern », Hamblin, C. L. 1970. Fallacies (Bungay, Suffolk : Methuen), 9-49

Hansen, Hans V. \& Robert C. Pinto. (eds). 1995. Fallacies: Classical and Contemporary Reading

(University Park : Pennsylvania State University Press), 98-105

Hoagland, John (ed). The Philosophy of Argument (Newport News : Vale)

Leff, Michael \& Robert Terrill. 1995. «The Polemicist as Artist : W. E. B. DuBois' Of Mr. Booker T. Washington and Other ", Jackson, Sally et al. (eds). Argumentation and Values: Proceedings of the $9^{\text {th }}$ AFA/SCA Conference on Argumentation (Annandale, VA : SCA), 230-236

Perelman, Chaim \& Lucie Olbrechts-Tyteca. 1992 (5éd.). Traité de l'Argumentation. La nouvelle rhétorique (Bruxelles : Editions de l'Université de Bruxelles)

Van Eemeren, Frans H. \& Rob Grootendorst. 1995. « Argumentum ad Hominem: A PragmaDialectical Case », Hansen and Pinto, 223-228

Van Eemeren, Frans \& Rob Grootendorst. 1995. «Perelman and the Fallacies », Philosophy and Rhetoric 28, 122-133

Walton, Douglas. 1998. Ad Hominem Arguments (Tuscaloosa : University of Alabama Press)

\section{NOTES}

1. Au sujet $d u$ «traitement standard» des arguments fallacieux, voir Hamblin 1970 : 9-49, Hansen \& Pinto 1995 : 98-105, et en rapport avec la rhétorique, Amossy 1999 : 14-18.

2. Hamblin $1970: 12$, cité par Hansen \& Pinto $1995: 97$

3. van Eemeren \& Grootendorst 1995 (Hansen \& Pinto, 223-228)

4. Sur cette différence, voir, par exemple, van Eemeren \& Grootendorst 1995 : 122-133.

5. Sur les variations de la rhétorique classique, voir Conley $1990: 4-25$.

6. Pour une analyse rhétorique plus complète et plus élaborée de cet essai, voir Leff \& Terrill 1995 : 230-236. 


\section{RÉSUMÉS}

La conception de Perelman sur le rôle des personnes dans l'argumentation est l'une des marques les plus caractéristiques de sa rupture avec les hypothèses cartésiennes sur le raisonnement. Alors que le paradigme rationaliste cherchait à minimiser ou à éliminer les considérations personnelles comme étant dilatoires et non pertinentes dans ce cadre, Perelman insiste sur le fait que l'argumentation met inévitablement l'accent sur les personnes spécifiques impliquées dans l'argumentation et que la relation entre le locuteur et ce qui est dit est toujours pertinente et importante. En adoptant cette position, Perelman ressuscite implicitement la conception classique de la preuve par le caractère (argument par l'ethos ou « ethotique »). Mais, bien que la Nouvelle Rhétorique consacre une large place au débat sur l'acte et la personne dans l'argumentation, elle n'accorde pas beaucoup de réflexion au concept classique et mélange différentes approches à l'intérieur de la tradition. Le résultat est que Perelman traite le rôle du locuteur dans l'argumentation uniquement en référence à des techniques abstraites et ne prend pas en compte l'importance de l'examen des cas particuliers pour éclairer la compréhension du fonctionnement de l'argument ethotique en situation dans le contexte complexe de son utilisation effective. Par conséquent, la prise en compte par Perelman du rôle de la personne dans l'argumentation doit être complétée par la référence à des études de cas et c'est dans ce but que j'étudie l'argument ethotique dans le célèbre essai de W. E. B. DuBois «Sur M. Booker T. Washington et autres ».

Perelman's view of the role of persons in argument is one of the most distinctive features of his break with Cartesian assumptions about reasoning. Whereas the Rationalist paradigm sought to minimize or eliminate personal considerations by dismissing them as distracting and irrelevant, Perelman insists that argumentation inevitably does and ought to place stress on the specific persons engaged in an argument and that the relationship between speaker and what is spoken is always relevant and important. In taking this position, Perelman implicitly revives the classical conception of proof by character (ethos or "ethotic" argument), but despite an extended discussion of act and person in argument, The New Rhetoric does not give much consideration to the classical concept and confuses differing approaches to it within the tradition. The result is that Perelman treats the role of the speaker in argument only by reference to abstract techniques and does not recognize the importance of examining particular cases in order to thicken understanding of how ethotic argument works in the complex, situated context of its actual use. Consequently, Perelman's account of the role of persons in argument should be supplement by reference to case studies, and to that end, I consider ethotic argument in W.E.B. Dubois' famous essay "Of Mr. Booker T. Washington and Others."

\section{INDEX}

Keywords : ad hominem argument, ethos, ethotic, interaction

Mots-clés : argument ad hominem, ethos, ethotique, interaction

\section{AUTEURS}

\section{MICHAEL LEFF}

Université de Memphis 